\title{
Evaluación en contextos indígenas: el papel de las ideologías en la pertinencia cultural del proceso
}

\author{
Evaluation in indigenous contexts: \\ the role of ideologies in the cultural relevance of the process
}

Yeny Vargas Iturra

Universidad Católica de Temuco, Chile. yenyvargasiturra@gmail.com

\begin{abstract}
RESUMEN
El siguiente ensayo teórico se estructura con el propósito de comprender el papel de las ideologías curriculares en la evaluación de contextos indígenas latinoamericanos. El problema que está a la base de este texto es la evidente persistencia a través del tiempo de componentes ideológicos distantes del contexto local y excluyente de la cultura indígena, situación que se acrecienta a través de la inclusión de elementos instrumentalistas propios del sistema neoliberal. Estas condicionantes han posibilitado la pérdida gradual de elementos culturales y brechas en cuanto a rendimiento escolar. Puesto que finalmente la evaluación instrumental y de alta consecuencias tensiona hacia la asimilación y mide el grado en el que este proceso se manifiesta en el estudiante indígena, invisibilizando sus conocimientos culturales y capacidades. Esto ocurre al no relacionarse la cultura escolar con el lenguaje y racionalidad indígena, hecho que además propicia desajustes en los estudiantes indígenas que deben resignificar sus códigos culturales de modo de transitar por los niveles académicos de forma "exitosa".

En suma, este ensayo discute las formas e implementaciones evaluativas y su relación con la cultura. Desde la perspectiva metodológica, el presente texto considera un análisis bibliográfico, estableciendo relaciones y efectos en función de una descripción general de las ideologías y modelos evaluativos que subyacen en la educación latinoamericana.
\end{abstract}

Palabras claves: Ideología neoliberal, ideología crítica, evaluación instrumental, evaluación dialógica.

\begin{abstract}
The following theoretical essay study is structured with the purpose of understanding the role of curricular ideologies in the evaluation in Latin American indigenous contexts. The problem that lies at the base of this text is the evident persistence over time of ideological components, distant from the local context and excluding indigenous culture, a situation that is increased through the inclusion of instrumental elements of the neoliberal system. These conditions have made possible the gradual loss of cultural elements and gaps in terms of school performance, since finally the instrumental evaluation and high consequences, stresses towards assimilation and measures the degree to which this process manifests itself in the indigenous student. Since the contents and forms of evaluation are not related to the indigenous culture, language and rationality, propitiating an imbalance in the indigenous student that must be adapted to the school culture, resignifying the cultural codes, so as to be able to travel through the academic levels in a "successful" way.

In this way, the evaluation does not reflect the abilities of the students, but rather their ability to understand the majority culture. From the methodological perspective, this study considers a bibliographic analysis, establishing relationships and effects based on a general description of the ideologies and evaluative models that underlie Latin American education.
\end{abstract}

Key words: Neoliberal ideology, critical ideology, instrumental evaluation, dialogical evaluation. 


\section{INTRODUCCIÓN}

La evaluación en contextos indígenas se puede entender de diversas maneras de acuerdo con las necesidades, propósitos u objetivos que se dan en un contexto histórico específico (Guerrero y Benavent, 2002). Algunas definiciones presentan una orientación meramente cuantitativa de control y medición, mientras que otras están orientadas a fines formativos y de emancipación (Martínez, 2012). La evaluación educativa estará asociada a una teoría institucional y a la forma masificada en la que se desarrollan los procesos de valoración de acuerdo con la época y el contexto (Mora, 2004). En este sentido, este ensayo discute acerca de la evaluación educativa en el contexto latinoamericano de la época postmoderna. El texto se sitúa desde la teoría social crítica, el enfoque de educación intercultural y la pedagogía emancipadora de Freire (1980). Haciendo evidente en la discusión como problema central la tendencia Latinoamérica hacia enfoques instrumentales de evaluación y modelos ligados a la acción y consecución de metas (Del Pino, 2014). Estas formas de pensar la educación y la evaluación generan la exclusión de los elementos culturales y sociales propios de los pueblos indígenas, los cuales están fundamentados en una racionalidad y cosmovisión opuesta a la visión instrumental frecuente en la escuela (Roura, 2017; Quilaqueo, Quintriqueo, Torres y Muñoz, 2014). Desde esta perspectiva es que este texto se propone como objetivo, analizar las ideologías curriculares que intervienen y tensionan el concepto de evaluación en contextos con diversidad social y cultural, reconociendo a estas formas ideológicas como la principal limitante para la inclusión de modelos transformadores y culturalmente pertinentes.

En consideración a esto, el texto desarrolla la idea de la evaluación como un instrumento que desde su base ideológica puede generar exclusión o trasformación (Treviño, Morawietz, Villalobos y Villalobos, 2017; Guerrero y Benavent, 2002; Ferrada y Flecha, 2008). Para fundamentar este posicionamiento, se discutirá sobre la base de dos grandes perspectivas ideológicas, que influencian la cultura escolar en la época post moderna: la ideología neoliberal y la ideología crítica (Gazmuri, 2017; Pozo, 2014). Constatándose a partir de ellas la generación de enfoques evaluativos tanto instrumentales (técnicas ligadas al control externo) como dialógicos (asociados a la co-construcción) (Mora, 2004). Este artículo y las ideas que en él se discuten, sistematizarán las dimensiones de la evaluación que son intervenidas por la ideología.

La metodología utilizada en este texto es el análisis bibliográfico en base a 31 artículos científicos de diversa indexación: Redalyc, Scielo, Web of Science, Google Scholar y Science Direct. Junto con ello se realiza una revisión de 1 tesis asociada a la temática y literatura clásica de corriente hispanoamericana y anglosajona, derivadas de la sociología y pedagogía críticas. La revisión se realiza en base a las palabras claves: ideología neoliberal, ideología crítica, evaluación dialógica, evaluación instrumental. Dando como resultado los siguientes apartados que se discutirán a continuación: Evaluar y educar en el contexto latinoamericano: la situación indígena; influencias de la ideología neoliberal en la evaluación educativa; ideología crítica y evaluación en contexto indígena, desde una perspectiva dialógica.

\section{EVALUAR Y EDUCAR EN EL CONTEXTO LATINOAMERICANO: LA SITUACIÓN INDÍGENA}

La tendencia de los sistemas educativos latinoamericanos y sus correspondientes modelos evaluativos es a replicar en los distintos países sin mayor contextualización las 
ideas heredadas de la cultura occidental (Del Pino, 2014; Jaksic y Rengifo, 2006). Esta tendencia a aplicar modelos externos se ha producido históricamente por el paradigma de la homogenización que se instauró en la América Latina colonial, ideas sobre una jerarquía determinada que adquirieron un papel central en la demarcación de las líneas de inclusión y exclusión social, segregando a la población indígena (Quijano, 2015). La visión homogeneizadora se mantiene en las estructuras sociales pese a los cambios políticos y normativos a nivel internacional que validan la diversidad cultural y la resguardan (Convenio 169 OIT, UNICEF, 1989; OCDE, 2006). Esto producto de instituciones sociales como la escuela que reproducen las ideologías de las clases dominantes, asumiendo sin reflexión y crítica la exclusión como parte de su estructura (Apple, 2008). Producto de estos factores sociales, históricos e ideológicos el estudiante que proviene de un entorno indígena es aislado de sus propios medios de producción de significado, vinculados a su experiencia familiar y cultural, la que no es considerada en el contexto de la escuela (Freire, 1980; Lagos, 2015; Giroux y McLaren, 2011). Estos elementos de segregación explicitados a través de la pedagogía y sociología crítica son los que han mantenido la asimetría educativa, existiendo una tendencia hacia la invisibilización de la cultura del indígena, situación que caracteriza los sistemas educativos latinoamericanos (ONU, 2007; Bengoa, 2007). Desde la descripción del sistema educativo, surge la pregunta: ¿Cuál es la posición de la evaluación en estos sistemas educativos? y ¿cuál es la repercusión que estos tienen en los estudiantes indígenas?

La posición de la evaluación es central, puesto que dirige de modo implícito el desarrollo de los sistemas educacionales, siendo el modelo seleccionado para su implementación el condicionante de los procesos de enseñanza y aprendizaje que se practican en los diferentes niveles educativos (Castillo, 2002). Por lo tanto, en el marco de instituciones homogeneizadoras como las descritas en Latinoamérica, la evaluación es planteada como un instrumento que no utiliza la diversidad como recurso, sino que al contrario contribuye desde su perspectiva unitaria a la asimilación cultural (Treviño et al., 2017; Baeza, 2006; Gimeno, Pérez y Gómez, 1996).

La evaluación genera asimilación al prevalecer modelos técnicos, orientados a la acción y consecución de metas, en un paradigma de la efectividad y mejora escolar (Del Pino, 2014). A su vez, mediante este modelo técnico, las evaluaciones que se desarrollan presentan altas consecuencias y son utilizadas para otorgar premios y sanciones regulando el traspaso de etapas en el sistema educativo (Hamilton, Stecher y Klein, 2002). Junto con esto se afectan las oportunidades de los estudiantes, pues son utilizadas para procesos de selección (Martínez, 2012; Ravela, 2001). El problema de este enfoque técnico radica en la habilidades y contenidos que mide, ya que, según lo expuesto por Treviño et al. (2017), la visión técnica ha contribuido al desarrollo de evaluaciones que miden el grado de aculturación o asimilación del estudiante indígena, en lugar de entregar información pertinente sobre sus procesos de construcción de conocimiento.

De esta forma los alumnos(as) indígenas latinoamericanos deben adaptarse a la evaluación homogénea considerada "objetiva", para superar los hitos establecidos en los sistemas oficiales (Bruzual y Sánchez, 2014), hitos que representan a la cultura dominante. En este sentido, la evaluación es un mecanismo que ha respaldado y gatillado la asimilación de los jóvenes y niños(as) indígenas que se exponen frecuentemente a la cultura escolar unitaria y homogeneizante y deben transitar por la escuela comprendiendo sus contenidos y respondiendo sus evaluaciones (Walsh, 2005; Arriagada, 2010). 
Los análisis que se han hecho al sistema general educativo latinoamericano en consideración a la inclusión de la cultura indígena arrojan la necesidad de avanzar hacia un desarrollo que contemple la equidad y la pertinencia (López, 1996; Mato, 2008; Loncón, 2014; Lagos, 2015; Walsh, 2005). Del mismo modo los organismos internacionales han comprometido iniciativas tendientes a fortalecer las culturas originarias desde la educación (UNESCO, 2007; OCDE, 2006), motivando a los gobiernos a desarrollar leyes y políticas públicas en esta línea. Sin embargo, se mantienen en el sistema lógicas que no permiten que se desarrollen modificaciones profundas, estas radican en la función ideológica de los currículum que se instauran en el ámbito educacional (Shiro, 2007).

Figura 1. Características de la evaluación en contextos latinoamericanos para estudiantes indígenas, consecuencias y tipo de medición

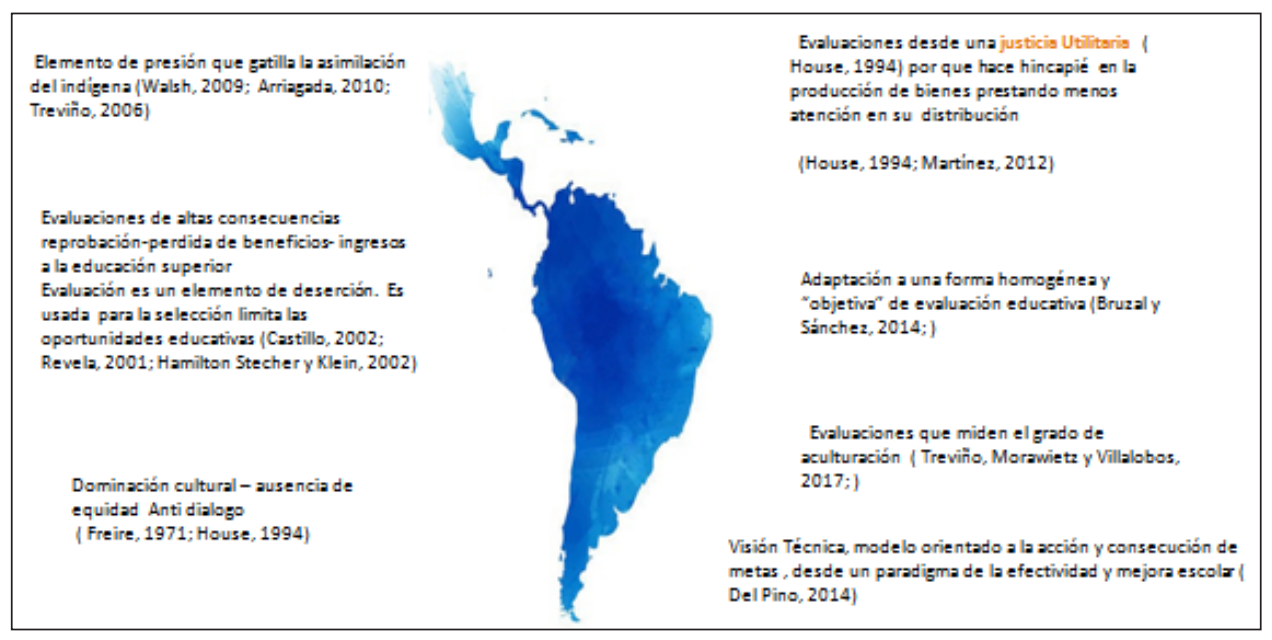

Fuente. Elaboración propia en función a los diversos autores citados en el apartado (Walsh, 2005; Arriagada, 2010; Treviño, 2006; Castillo, 2002; Revela, 2001; Hamilton, Stecher y Klein, 2002; Freire, 1980; House, 1994;

Martínez, 2012; Bruzual y Sánchez, 2014; Del Pino, 2014).

\section{INFLUENCIAS DE LA IDEOLOGÍA NEOLIBERAL EN LA EVALUACIÓN EDUCATIVA}

La ideología puede ser definida como un conjunto de ideas, pensamientos y prácticas que están relacionadas con un sistema general que comprende el ámbito político, social, económico, científico y tecnológico (Prieto, 1979). Con relación a esto, una ideología curricular tendrá ideas y concepciones sobre el tipo de ser humano que pretende formar, la sociedad que desea construir y las características de la escuela (Apple, 2008). En este sentido, la evaluación es un componente más de la ideología, ya que se asocia a una idea sobre educación y es considerada un medio para promover los objetivos seleccionados en función a este ideario establecido (Pórtela, 2004). 
Los constructos ideológicos se pueden clasificar de distintas formas, para esta discusión consideraremos dos, la ideología crítica comunicativa que desarrollo modelos evaluativos dialógicos y la ideología neoliberal que desarrolla modelos técnicos e instrumentales de evaluación (Apple, 2008; Giroux y Mclaren, 2011).

Asociado a la ideología y la evaluación, es importante lo ya señalado sobre la historia de la educación latinoamericana, en cuanto a la exclusión de la cultura indígena (García, 2014; Guerrero, 2012). Esta exclusión surgió desde la imposición de un consenso ideológico y hoy se mantiene producto de la ideología neoliberal, la cual vincula el sistema económico, político, social y educativo. Seleccionando instrumentos y conocimientos en función de la economía global (García, 2014; Guerrero, 2012; Shiro, 2007; Giroux y Mc Laren, 2011; Habermas, 2001).

Las ideologías neoliberales son las que actualmente definen la cultura evaluativa que se desarrolla con mayor frecuencia en nuestro contexto latinoamericano (Gazmuri, 2017; Pórtela, 2004). A partir de esta ideología, la escuela será la responsable de impartir y distribuir un conocimiento de acuerdo con indicadores de desarrollo, a partir de formas institucionalizadas que configuran los roles y el tipo de evaluación que se desarrolla (Giroux y McLaren, 2011; Goodson, 2003). En consideración a lo planteado, la evaluación será un instrumento para medir rendimiento en función de indicadores preestablecidos por el mercado competitivo (Apple, 2008; Olive, 2011; Follari, 2006). Y el evaluador trasmitirá y validará el tipo de conocimiento impuesto a través de la evaluación que implementa (House, 1994; Giroux y McLaren, 2011).

Estas formas institucionalizadas y de raíz occidental que se han vuelto instrumentalizadas en relación a las necesidades del mercado, se mantienen sin mayor cuestionamiento, producto de categorías creadas a través de los conocimientos seleccionados en los currículum escolares y la acción de intelectuales que las utilizan y las validan tanto en la academia como a través del ejercicio de la evaluación y enseñanza (Apple, 2008; Giroux y McLaren, 2011). Estos elementos no permiten el acceso a otras formas de evaluar y construir conocimiento, como las que nacen desde las culturas originarias.

En el caso indígena, las categorías predominantemente cientificistas y técnicas (Boix, 2004) que son seleccionadas, distribuidas y evaluadas por la escuela, son dilemáticamente opuestas a las construidas en las culturas originarias (Merino, Quilaqueo y Saiz, 2008). Esto se produce porque existe una distancia entre la racionalidad indígena y la racionalidad escolar, que se refleja en la forma de entender el mundo y las relaciones que se sucintan en el (Quilaqueo et al., 2014). La racionalidad indígena está caracterizada según Tobar (2009), por una visión integradora y una forma de generar conocimiento ligada a la práctica y las relaciones intersubjetivas con el todo. Estas formas de conocer y comprender el mundo no se comunican con las impuestas desde la escuela, caracterizadas por lógicas segmentadas, individualistas asociadas a la ideología dominante.

La prevalencia de estas lógicas neoliberales y la dificultad de generación de cambios en las instituciones educativas y su evaluación, generan consecuencias en el alumno(a) indígena; puesto que, asociada a esta ideología neoliberal se desarrolla un enfoque evaluativo instrumental que actúa desde lo orgánico y normativo facilitando con la acción evaluativa, la apropiación en los estudiantes indígenas de conocimientos y pautas culturales que legitiman la sociedad moderna y los apartan de sus propias formas culturales de generar conocimiento (Guerrero y Benavent, 2002; Carrillo, Araya y Henríquez, 2009). La evaluación se vuelve un instrumento de coerción que genera la asimilación a la cultura mayoritaria. 
Si bien la evaluación está llamada a ser un encuentro didáctico que facilite la aproximación progresiva a las representaciones y formas de construir el conocimiento que manifiesta el estudiante (Castillo, 2002; Mora, 2004), en el contexto monocultural de la mayoría de los sistemas educativos latinoamericanos, este encuentro didáctico no se efectúa. La escuela en lugar de ceñirse a la primera de sus funciones, que es la trasmisión de conocimientos para el desarrollo de todos los ciudadanos, se convierte desde su función ideológica en una constructora de perfiles de sujetos solicitados por el mercado (Giroux, 1990).

En contraposición a esta mirada técnica de la evaluación, occidentalizada y homogeneizante, surgen en Latinoamérica alternativas y formas de comprender la educación desde la igualdad educativa, que visualizan la cultura indígena como un recurso sobre el cual construir modelos justos de evaluación (Ferrada y Flecha, 2008; Cram y Mertens, 2016). Estos diseños tienen su base en una visión crítica sobre las lógicas que priman en el sistema educativo y mantienen las asimetrías, buscando transformarlas (Walsh, 2005; Freire, 1980). Nos referimos a las ideologías críticas y los modelos de evaluación dialógicos que se abordarán posterior al esquema que resume este aparatado.

Este esquema presenta como se define, la escuela, el conocimiento, el mundo indígena, y el evaluador en función de la ideología neoliberal. Junto con ello, los tres componentes que hacen que las lógicas hegemónicas se mantengan (categorías creadas - profesionales que las utilicen - saturación de la vida cotidiana).

Esquema 1. Influencia de la Ideología neoliberal en la evaluación educativa en contextos indígena

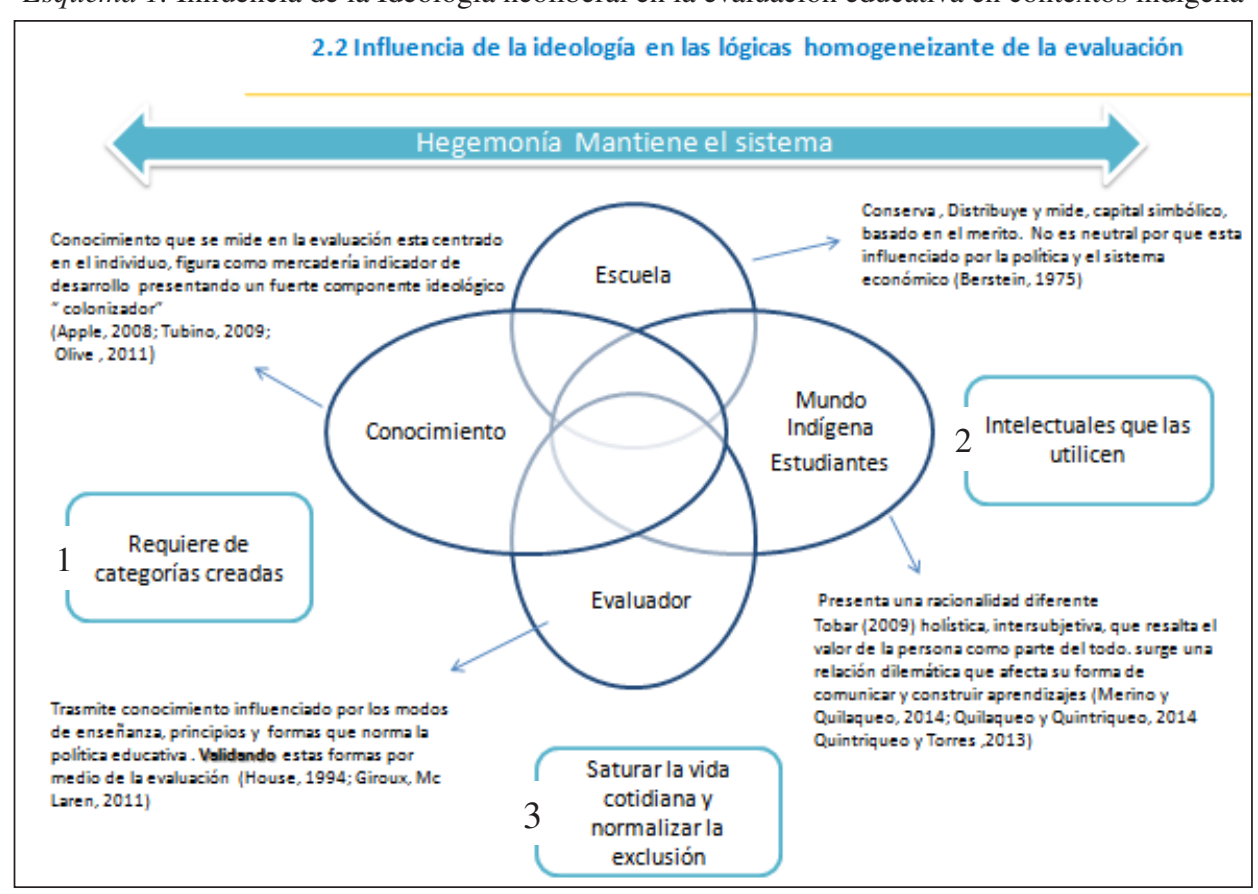

Fuente. Elaboración propia en función de los autores citados en el apartado (Apple, 2008; House, 1994; Giroux y McLaren, 2011; Tobar, 2009; Merino, Quilaqueo y Saiz, 2008; Quilaqueo et al., 2014; Tubino, 2005; Olive, 2011; Berstein, 1975). 


\section{IDEOLOGÍA CRÍTICA Y EVALUACIÓN EN CONTEXTO INDÍGENA DESDE UNA PERSPECTIVA DIALÓGICA}

Las ideologías críticas surgen como respuesta antagónica al pensamiento hegemónico que materializa las asimetrías sociales en distintos contextos (Apple, 2008; Pozo, 2014). Los planteamientos críticos sobre educación provienen desde diferentes ámbitos, como la sociología, pedagogía y antropología (Pozo, 2014). El objetivo que esta ideología educativa se plantea es formar ciudadanos críticos, comprometidos con la democratización de la educación (Bordieu, 1996; Gazmuri, 2017). Desde este planteamiento el conocimiento es una construcción en base a interpretaciones diversas y situadas, lo que permite el constante diálogo con distintas formas de construir conocimiento. Para la ideología crítica el objetivo de la educación es construir y contribuir a la conformación de una sociedad más justa, que valore la diversidad y la riqueza social (Mora, 2004). En cuanto a los contenidos, buscará desarrollar habilidades vinculadas con el análisis y la interpretación, siendo relevante para ello las experiencias y las vivencias de los estudiantes (Pozo, 2014; Freire, 1980).

En consideración a las características presentadas una evaluación desde la ideología crítica en contexto indígena significaría la valoración de sus experiencias y de sus formas particulares de construir conocimiento, lengua y cosmovisión (Schmelkes, 2013). En este sentido la evaluación buscaría comprender cómo se construyen activamente las categorías de significado en las comunidades y territorios indígenas, para permitirle a los estudiantes ubicarse en su historia y actuar en ella (Martínez, 2012). Considerando procesos negociación de todos los implicados, estos procesos serán comunicativos y su objetivo es lograr la concientización social y comunitaria (Ferrada, 2001; Guerrero y Benavent, 2002; Freire, 1980).

Las ideologías críticas se vinculan con la perspectiva dialógica de la evaluación, que persiguen los mismos fines emancipatorios, fundamentadas en un paradigma transformador y de igualdad educativa (Del Pino, 2014). Integrando a lo ya problematizado por la ideología crítica estrategias concretas para enfrentar la trasformación de los procesos educativos por medio de la participación igualitaria de todos los agentes implicados en los procesos de enseñanza y aprendizaje (Ferrada, 2001; Del Pino, 2016; Cram y Mertens, 2016). Los ejes del modelo dialógico de evaluación son: consenso, participación, altas expectativas, intersubjetividad y colaboración. Estos conforman la base para la construcción de conocimiento (Del Pino, 2014). En la línea de la evaluación dialógica, en contextos indígenas es relevante destacar la coherencia que todas las dimensiones (axiológica, ontológica, epistemológica y metodológica) deben tener con relación a la cosmovisión y racionalidad propia de las comunidades donde ha de construirse conjuntamente la evaluación (Cram y Mertens, 2016).

A partir de estas dimensiones se comprenderá la evaluación dialógica desde lo axiológico como un modelo asociado a la ética de la alteridad, lo que significa el desarrollo de un respeto cultural, la búsqueda de la justicia y la reducción de las desigualdades, que, en el caso indígena latinoamericano, están asociadas a la invisibilización de sus formas de construir conocimiento, cultura y lenguaje (Cram y Mertens, 2016; Bruzual y Sánchez, 2014; Schmelkes, 2013). Esta forma de concebir la evaluación repercute en el aula generando comunidades educativas donde existen espacios para la expresión del otro y su cultura a partir de la evaluación (Del Pino, 2016; Giroux y McLaren, 2011). Desde lo ontológico, el mundo no puede concebirse desde una lógica cerrada y herméticamente "objetiva", ya que la realidad tendrá múltiples opciones de acuerdo a las características de cada sujeto (Gazmuri, 
2017; Cram y Mertens, 2016). En este sentido la realidad para el mundo indígena es múltiple y holística, integral y no segmentada, una perspectiva de evaluación en contexto indígena debe considerar esta visión ontológica (Tobar, 2009). En cuanto al componente epistemológico, asociado al conocimiento, la perspectiva dialógica considera una reciprocidad epistémica (Del Pino, 2016) entendida como la posibilidad de alcanzar la equidad en la comunicación y construcción del conocimiento. Siendo conscientes del contexto de poder en el cual se enmarca la comunidad (Gazmuri, 2017; Giroux y McLaren, 2011). La dimensión metodológica se adhiere a la perspectiva constructivista, que busca la descolonización, basada en la relación crítica reflexiva a través de procesos interactivos que llevan a una concientización (Carbajosa, 2010; Cram y Mertens, 2016). En el caso indígena implica la autodeterminación educativa, el respeto recíproco y la co-construcción de conocimiento.

En virtud de lo discutido se puede señalar que el contexto inequitativo en el cual se sitúa la evaluación en Latinoamérica hace necesario reflexionar y replantearse las bases ideológicas que sustentan los sistemas formales, con la finalidad de dar cabida a acciones alternativas, las que surjan también desde lo ideológico, pero den cabida a las necesidades y características de todos los estudiantes, valorando el componente intersubjetivo de la construcción del conocimiento y su base cultural.

A continuación, la tabla 1 evidencia su impacto en cuanto a las dimensiones axiológicas asociadas a la ética, ontológicas vinculadas a la percepción de la realidad, epistemológicas relacionadas a la construcción de conocimiento y metodológicas que incorporan acciones evaluativas.

Tabla 1. Sistematización de la implicancia de las ideologías en la evaluación educativa

\begin{tabular}{|l|l|l|}
\hline $\begin{array}{l}\text { Dimensiones de } \\
\text { la evaluación }\end{array}$ & \multicolumn{1}{|c|}{$\begin{array}{c}\text { Ideologías neoliberales } \\
\text { Evaluación instrumental }\end{array}$} & \multicolumn{1}{c|}{$\begin{array}{c}\text { Ideologías críticas } \\
\text { Evaluaciones dialógicas }\end{array}$} \\
\hline Axiológico & $\begin{array}{l}\text { Ética de la justicia utilitaria: Homogénea } \\
\text { - objetiva (House, 1994; Martínez, 2012) }\end{array}$ & $\begin{array}{l}\text { Ética de la alteridad, respeto cultural, jus- } \\
\text { ticia social, reducción de las inequidades } \\
\text { (Bruzual y Sánchez, 2014; Cram y Mer- } \\
\text { tens, 2016; Schmelkes, 2013) }\end{array}$ \\
\hline Ontológico & $\begin{array}{l}\text { Realidad homogénea, derivada del con- } \\
\text { senso ideológico, que excluye cons- } \\
\text { trucciones diferentes (García, 2014; } \\
\text { Guerrero, 2012) }\end{array}$ & $\begin{array}{l}\text { La naturaleza de la realidad es un mun- } \\
\text { do con múltiples opciones (Cram y } \\
\text { Mertens, 2016; Gazmuri, 2017; Giroux } \\
\text { y McLaren, 2011) }\end{array}$ \\
\hline Epistemológico & $\begin{array}{l}\text { Conocimiento centrado en el individuo, } \\
\text { construido en contexto de poder y privi- } \\
\text { legio (Cram y Mertens, 2016) }\end{array}$ & $\begin{array}{l}\text { Reciprocidad epistémica (Del Pino, } \\
\text { 2016) El conocimiento es una construc- } \\
\text { ción en base a interpretaciones diversas } \\
\text { y situadas (Gazmuri, 2017; Cram y } \\
\text { Mertens, 2016) }\end{array}$ \\
\hline Metodológico & $\begin{array}{l}\text { Acciones evaluativas apegadas a la visión } \\
\text { técnica, modelo orientado a la acción y y } \\
\text { consecución de metas (Del Pino, 2014) }\end{array}$ & $\begin{array}{l}\text { Constructiva, que busca la de coloniza- } \\
\text { ción, ligada a un análisis estructural, ba- } \\
\text { sada en una relación crítica y reflexiva } \\
\text { (Carbajosa, 2010; Cram y Mertens, 2016) }\end{array}$ \\
\hline
\end{tabular}

Fuente. Elaboración propia en base a la sistematización se ha realizado en función de las dimensiones mencionadas por Cram y Mertens (2016) para el paradigma transformador. 


\section{DESAFÍOS PARA LA TRANSFORMACIÓN DE LA EVALUACIÓN EN CONTEXTO INDÍGENA}

Ante la inequidad de los curriculum y evaluaciones implementadas en nuestro contexto latinoamericano en relación con la cultura y significados indígenas, se hace necesario plantearse una reestructuración de los procesos en pos de responder a las características culturales de la población y al establecimiento de equidad y calidad que se requiere para todos los alumnos(as). En este sentido es necesario situarnos desde una ideología crítica que propicie la reflexión sobre las circunstancias en las cuales se implementan las acciones educativas en la actualidad y cómo impactan estas acciones en los estudiantes.

Para que la diversidad resulte en un enriquecimiento mutuo, es preciso que en los centros de enseñanza se abran espacios que permitan que esta se manifieste en condiciones de igualdad (Martínez, 2012; Carbajosa, 2010). El grado en que esto sea incorporado influirá como lo mencionan Juste et al. (2007) en la integración educativa y en la situación social de los alumnos, al evidenciarse una trasformación de sus procesos. Sin embargo, para que esto ocurra no solo debe modificarse en el sistema educativo sino también en el social (Rojo, 2003). Esto conlleva a un cambio en torno a las relaciones de poder que se gestan en la escuela y que muchas veces utilizan a la evaluación como instrumento de coerción (Giroux y McLaren, 2011).

En este sentido se hace necesario el desarrollo de una conciencia cultural crítica, como parte de los procesos formadores en todo orden: en aula, formación inicial docente y formación inicial profesional de distintas especialidades (Byram, 2005). Esta conciencia cultural crítica se vincula con las actitudes del saber ser (axiológico), reconocer las propias limitaciones y prejuicios para actuar sobre ellos. Las actitudes del saber (epistemológico), comprender características culturales externas y de interés. Junto con aquellas que regulan el hacer (metodológico) y permiten construir y evaluar conocimiento en base a las visiones y percepciones propias de una cultura determinada (Etxeberría, Karrera y Murua, 2010).

En términos axiológicos esta re-definición de los sistemas implica situarse desde la ideología crítica, significa pasar desde una justicia utilitaria de la evaluación como medio objetivo, a una ética de alteridad, que vincula la evaluación con la trasformación social (Bruzual y Sánchez, 2014; House, 1994). Una ética que también es de responsabilidad, asociada a la participación conjunta en los procesos de evaluación para conformar comunidades como espacios para la expresión del otro (Giroux y Mc Laren, 2011). En este sentido la evaluación desarrollaría una lógica de alteridad, reconociendo que el estudiante puede conquistar su realidad y desarrollarla creativamente (Bruzual y Sánchez, 2014; House, 1994). Esta ética de la alteridad provoca obligatoriamente un cambio en los roles tanto del profesor como de los alumnos y comunidad en general, quienes son llamados a romper los condicionamientos del enfoque técnico, para hacerse conscientes del otro, como seres que tienen infinitas posibilidades de ser (Bruzual y Sánchez, 2014). Para todo ello la conciencia cultural y la reflexión crítica son dos elementos primordiales que pueden empoderar y generar cambios en el sistema formal y de esta forma vencer el anti-diálogo crónico que la escuela ha mantenido con la cultura indígena. 
Estudios Pedagógicos XLV, N² 2: 197-208, 2019

EVALUACIÓN EN CONTEXTOS INDÍGENAS: EL PAPEL DE LAS IDEOLOGÍAS EN LA PERTINENCIA CULTURAL DEL PROCESO

El siguiente esquema muestra los cambios y transiciones que significa adoptar una visión ideológica que integre elementos culturales en los procesos evaluativos.

Esquema 2. Desafíos para la evaluación en contextos indígenas



Fuente. Elaboración propia en base a los contenidos del apartado y autores (Gazmuri, 2017; Pozo, 2014; Freire, 1980; Del Pino, 2016; Apple, 2008; Ferrada, 2001; Schmelkes, 2013).

\section{REFERENCIAS BIBLIOGRÁFICAS}

Apple, M. (2008). Ideología y Currículo. Madrid, España: Ediciones Akal.

Baeza, R. (2006). Globalización y Homogenización Cultural. Sociedad Hoy, (10), 9-24.

Bengoa, J. (2007). La emergencia indígena en América Latina. Santiago, Chile:

Fondo de Cultura Económica.

Bernstein, B. (1975). Class and pedagogies: Visible and invisible. Educational studies, 1(1), 23-41.

Bourdieu, P. (1996). Cosas dichas. Barcelona: Gedisa.

Boix, R. (2004). Pluralismo epistemológico, entre el sueño objetivista y la inconmensurabilidad de paradigmas. Utopía y Praxis Latinoamericana, 9(24), 51-65.

Bruzual, A., y Sánchez, J. (2014). De la evaluación instrumental a la evaluación desde la alteridad. Revista multidisciplinaria del consejo de investigación de la universidad de oriente. 26(3), 335-342.

Byram, M. (2005). Intercultural competence and the politics of foreign language education. Cultura, Interculturalidad y Didáctica de la lengua y la literatura, 25-34. 
Castillo, S. (2002). Compromisos de la evaluación educativa. Madrid, España: Pearson.

Carbajosa, D. (2010). Debates desde paradigmas en la evaluación educativa. Perfiles educativos, 33(32), 183-192.

Carrillo, M. F., Araya, C. C., \& Henríquez, S. S. (2009). Enfoques y concepciones curriculares en la Educación Parvularia. Revista de Pedagogía, 30(86), 47-70.

Cram, F., y Mertens, D., (2016). Negotiating solidarity between indigenous and transformative paradigms in evaluation. Evaluation Matters-He Take Tō Te Aromatawai, 2, 161-189.

Del Pino, M. (2016). Comunidad de Evaluación: protagonistas en las decisiones evaluativas. Educere, 20(65), 61-71.

Del Pino, M. (2014). Caracterización Teórica del Modelo de Evaluación Comunicativa. Revalue, 3(1).

Etxeberría, F., Karrera, I., Murua., H., (2010). Competencias interculturales del profesorado con alumnado inmigrante vasco. Revista interuniversitaria de formación del profesorado. 13(4), 79-94.

Ferrada, D. (2001). Currículum Crítico Comunicativo. Barcelona: El Roure.

Ferrada, D., y Flecha, R., (2008). El modelo dialógico de la pedagogía: un aporte desde las experiencias de comunidades de aprendizaje. Estudios pedagógicos (Valdivia), 34(1), 41-61.

Follari, R. (2006). Modificaciones epistemológicas actuales, oferta educativa y organización curricular. Revista RS, 2(56), 171-180.

Freire, P. (1980). Pedagogía del Oprimido. México: Siglo XXI.

García, M. (2014). Educar en la sociedad contemporánea, hacia un nuevo escenario educativo. Convergencia, 20(62), 199-220.

Gazmuri, R. (2017). Ideologías Curriculares en el debate y negociación del currículum chileno de Historia, Geografía y Ciencias Sociales. Estudios Pedagógicos (Valdivia), 43(1), 157-169.

Gimeno Sacristán, J. y Pérez Gómez, A. (1996). Comprender y trasformar la enseñanza. Madrid: Morata.

Guerrero, E. (2012). Un enfoque reflexivo del proceso de evaluación educativa. Reieg, (3), 102-117.

Guerrero, P., y Benavent, G., (2002). El modelo dialógico en la enseñanza aprendizaje, hacia una pedagogía de la interculturalidad. Asele, actas XIII, Centro Virtual Cervantes.

Giroux, H. (1990) Los profesores como intelectuales. Barcelona: Paidós.

Giroux, H., y McLaren, P. (2011). Sociedad Cultura y Educación. Buenos Aires: Ediciones Miño y Dávila.

Goodson, I. (2003). Estudios del Currículum. Buenos Aires: Amorrortu.

Habermas, J. (2001). Democracia, inclusión del otro y patriotismo constitucional desde la ética del discurso. Revista Derecho y Ciencia política, (3).

Hamilton, L. S., Stecher, B. M., y Klein, S. P. (2002). Making sense of test-based accountability in education. Santa Mónica: Rand Corporation.

House, E., R. (1994). Evaluación Ética y Poder. Madrid: Morata.

Jaksic, I., Rengifo, F. (2006). Historia política de Chile, 1810- 2010. Santiago, Chile: Fondo de Cultura Económica.

Juste, R. P., Rupérez, F. L., Peralta, M. D. F., y Fernández, P. M. (2007). Hacia una educación de calidad. Gestión, instrumentos y evaluación. Avances en supervisión educativa: Revista de la Asociación de Inspectores de Educación de España, (5), 15.

Lagos, C. (2015). El programa de educación intercultural bilingüe y sus resultados ¿perpetuando la discriminación? Pensamiento Educativo, 52(1), 84- 94.

Lomas, C. (2016). Lo lingüístico es político. Cuadernos de pedagogía, 465, 56-61.

Loncón, E. (2014). Educación y Cultura: ¿Podremos llegar a ser una sociedad intercultural? En B. Carimán (ed) ¿Chile indígena? Desafíos y oportunidades para un nuevo trato (109-120). Santiago: El Desconcierto- Programas de Derechos Indígenas Fundación Chile 21.

López, L. (1996). La diversidad étnica, cultural y lingüística latinoamericana y los recursos humanos que la educación requiere. Revista Iberoamericana de Educación, (13), 47-98. 
Mansilla, J., Llancavil, D., Mieres, M., Montanares, E. (2016). Instalación de la escuela monocultural en la Araucanía, 1883-1910: dispositivos de poder y sociedad mapuche. Educación y Pesquisa, 42(1), 213-228.

Martín, P. A. (2007). Evaluación formativa y su repercusión en el clima del aula. Revista de Investigación educativa, 25(2), 389-402.

Martínez, F. (2012). El futuro de la evaluación educativa. Sinéctica, (40), 2-11.

Mato, D. (2008). No hay saber "universal", la colaboración intercultural es imprescindible. Alteridades, 18(35), 101-116.

Merino, M. E., Quilaqueo, D., y Saiz, J. L. (2008). Una tipología del discurso de discriminación percibida en mapuches de Chile. Revista signos, 41(67), 279-297.

Mora, A. I. (2004). La evaluación educativa: concepto, períodos y modelos. Actualidades Investigativas en Educación, 4(2) 1-27.

OCDE, P. (2006). Marco de la evaluación. Conocimientos y habilidades en Ciencias, Matemáticas y Lectura.

Olive, L. (2011). Interculturalismo y justicia social. México: Editorial UNAM.

Pórtela, H. (2004) La racionalidad técnica, una huella que condiciona el sentido de la formación en los procesos educativos. Revista Ired, (1).

Pozo, G. (2014) ¿Cómo decolonizar el saber? El problema del concepto de interculturalidad. Polis, (38), 1- 15.

Prieto, J. M. L. (1979). Vigencia del pensamiento de Gramsci. El Basilisco: Revista de materialismo filosófico, (6), 73-83.

Quezada, D. (2017). Importancia del enfoque trasformador. Revista reflexiones, 96(1), 57-73.

Quilaqueo, D., Quintriqueo, S., Torres, H., \& Muñoz, G. (2014). Saberes educativos mapuches: aportes epistémicos para un enfoque de educación intercultural. Chungará (Arica), 46(2), 271-284.

Quijano, A. (2015). Colonialidad de poder, y clasificación social. Contextualizaciones latinoamericanas, 3(5), 1- 33.

Ravela, P. (2001). Los próximos pasos: ¿cómo avanzar en la evaluación de aprendizajes en América Latina? PREAL.

Roura, M. (2017). Evaluación dialógica en entornos MOOC. Tesis Doctoral. UNED.

Rojo, L. (2003). El análisis crítico del discurso: fronteras y exclusión social en los discursos racistas. En Manual para las ciencias sociales. (pp. 157-189). Barcelona, España: Editorial UOC.

Schmelkes, S. (2013). Experiencia en México. Hacia una evaluación con enfoque intercultural. https://tarea.org.pe/wp-content/uploads/2015/12/Tarea89_60_Sylvia_Schmelkes.pdf

Shiro, M. (2007). Curriculum theory: conflicting visions and enduring concerns. Thousand Oaks: Sage Publications.

Treviño, E. (2006). Evaluación de los aprendizajes de los estudiantes indígenas en América Latina. Desafíos de Medición e interpretación en contextos con diversidad cultural y desigualdad social. Revista mexicana de investigación educativa, 11(28), 225-268.

Treviño E., Morawietz L., Villalobos C. y Villalobos, E. (2017). Educación Intercultural en Chile, Experiencias, Pueblos y Territorios. Ediciones Universidad Católica de Chile. Santiago de Chile.

Tobar. M. (2009, 25-28 agosto). Interculturalidad y Epistemología. Seminario Nacional de investigación en educación, pedagogía y formación docente. Medellín: Universidad de Antioquia Colombia.

Tubino, F. (2005). La praxis de la interculturalidad en los estados nacionales latinoamericanos. Cuadernos Interculturales, 3(5), 83- 95.

UNESCO. 2007. Organización de las Naciones Unidas para la Educación la Ciencia y la Cultura Declaración de Naciones Unidas sobre los Derechos de los pueblos Indígenas. Santiago: Oficina Regional de Educación para América Latina y el Caribe. OREALC/UNESCO. 2007.

Walsh, C. (2005). Interculturalidad, conocimientos y decolonialidad. Signo y pensamiento, 24(46), 39-50. 